\title{
Behavioral and exercise interventions for sleep dysfunction in the elderly: a brief review and future directions
}

\author{
Amy Gencarelli ${ }^{1}$. Anne Sorrell ${ }^{1}$. Cassandra M. Everhart ${ }^{2} \cdot$ Taylor Zurlinden $^{1} \cdot$ D. Erik Everhart ${ }^{1}$ (I)
}

Received: 28 August 2020 / Revised: 26 January 2021 / Accepted: 19 February 2021 / Published online: 25 February 2021

(C) The Author(s), under exclusive licence to Springer Nature Switzerland AG part of Springer Nature 2021

\begin{abstract}
Purpose The impact of sleep-related changes and disorders in the geriatric populations are of utmost concern due to health consequences and increased risk of injury as well as injuring others as a result of poor sleep. The purpose of this paper is to provide a brief review of the current state of the literature with regard to sleep, aging, common non-pharmacological interventions, and the potential use of exercise in combination with behavioral interventions.

Methods Initially, this manuscript focuses on a brief (nonsystematic) review of sleep parameters and physiology that are associated with the aging process. Subsequently, information regarding sleep disorders in the elderly in general, and insomnia in particular are discussed. Last, a brief review of current recommended interventions is provided.

Results The current major nonpharmacological interventions are described including Cognitive Behavioral Therapy for Insomnia (CBT-I). The potential use of exercise as a safe intervention for poor sleep is discussed. Finally, a call is made for increased research that examines the combination of traditional behavioral interventions with exercise.
\end{abstract}

Keywords Elderly $\cdot$ Insomnia $\cdot$ Sleep $\cdot$ Behavioral intervention $\cdot$ Exercise

\section{Introduction}

Sleep is an essential biobehavioral state that supports a wide range of systems, including but not limited to immune function, metabolism, cognition, and emotion regulation [1]. Untreated insomnia and other sleep disorders are considered global health epidemics [2] and can have major health and behavioral health consequences (e.g., cardiometabolic disease, impaired cognitive functioning, and increased risk of psychiatric disorders; [1]). Additionally, insufficient sleep is associated with an increased risk of accidents [3]. There is also data supporting a relationship between aging and increased risk of injury to self or others as a result of sleep dysfunction (e.g., Noh et al. [4] found a relationship between sleep duration and falling among the elderly). Advancement of the understanding of this relationship is important, particularly given that sleep quality often diminishes with age [5]. In light of these issues, research that pertains to aging,

D. Erik Everhart

everhartd@ecu.edu

1 Department of Psychology, East Carolina University, Greenville, NC 27858, USA

2 Honors Tutorial College, Ohio University, Athens, OH 45701, USA sleep changes and dysfunction, and efficacious interventions is a priority. Moreover, it is pertinent to understand the potential relationship between sleep and aging with exercise, which also tends to decline with age [6]. The purpose of this paper is to provide a brief review of the literature with regard to sleep, aging, common interventions, and the potential use of exercise in combination with behavioral interventions. A brief review of sleep parameters and physiology in the elderly is provided. Information regarding sleep disorders in the elderly, in general, and insomnia in specific is discussed. The current major nonpharmacological interventions are described as well as the potential use of exercise as a safe intervention for poor sleep is discussed. A call for research that examines the combination of traditional behavioral interventions with exercise is provided.

\section{Sleep and aging}

\section{Physiology}

Sleep quantity and quality tend to decrease with age [5] with incidence rates for insomnia in otherwise healthy elderly individuals ranging from $5 \%$ [7] to upwards of $12-25 \%$ [8]. Other studies suggest that this rate can be as high as 50\% [9]. This 
quantity/quality decrease appears to relate to the theory that sleep need is strongly modulated by the amount of brain plasticity during prior wake states (e.g., the more the brain learns and adapts to changing environments, the more sleep is needed [10]). Further, it has been hypothesized that poor sleep in the elderly may be caused by a chronic decrease in sleep need as a result of reduced opportunity to learn as well as decreased exposure to novel experiences in combination with changes in health and neural circuitry as it relates to sleep regulation [10]. Healthy aging typically results in an advancement of circadian rhythm (e.g., earlier bed and wake times) [11], with reductions in total sleep time, sleep efficiency, slow wave sleep, REM sleep, and REM latency [12]. It has also been found that sleep latency, NREM 1, NREM 2, and wake after sleep onset significantly increase with age [12]. Some researchers hypothesize that older adults need less sleep overall as they age (reduced sleep-need) while others suggest that aging reduces the ability to obtain restorative sleep (reduced sleep-ability), but research utilizing behavior and polysomnography (PSG) outcomes have found it difficult to disentangle the two views [13]. Overall, research favors the need for sleep in older age, and the issues in sleep quality and duration are thought to be due to cortical thinning and amyloid deposition, weakening the brain's ability to produce restorative sleep [13]. An additional factor to consider with measuring sleep across the lifespan are the alterations of sleep spindles, which were the original features of sleep described in electroencephalogram (EEG) recordings that reflected the waxing-and-waning nature of the $7-15 \mathrm{~Hz}$ spindle oscillation of about one second during NREM sleep [14]. It is important to note that while the strength of having objective EEG data was introduced to compliment subjective data at the time, there are paired limitations in terms of the "first-night effect," particularly in studies with only one night of EEG data. The first-night effect has been found to influence alpha-wave activity (e.g., enhanced wakepromoting system during sleep latency [SL]) and stage changes from alpha to theta across nights [15]. In general, the first half of the sleep cycle is composed of three NREM stages in which electrical activity across the brain decreases in frequency and increases in amplitude with stage progression; representing a reduction in overall neural activity but an increase in coordination among neurons or enhanced oscillation [1]. Stage 2 of sleep contains the presence of K-complexes (i.e., large-amplitude, rapidly fluctuating bursts of brain activity) and sleep spindles (i.e., $12-15 \mathrm{~Hz}$ of oscillating signals lasting $0.5-2 \mathrm{~s}$ ), which are hypothesized to support memory consolidation and filter sensory input [1]. With age, the density of K-complexes and sleep spindles decrease [16]. At older ages, spindle density decreases are most pronounced at both the frontal and occipital cortices, potentially relating to progressive neurodegenerative changes that preferentially affect these cortical areas with aging $[17,18]$. These spindle changes correlate with changes in sleep architecture and cognition although there is a large gap in the understanding of the neurophysiological changes that cause these declines in spindling as individuals age [19]. Researchers currently attempting to explain such changes discuss both decreases in cortical volume (i.e., the "shrinking brain phenomenon") as well as decrease in gray and white matter among various regions of the brain $[20,21]$. Toward the end of NREM sleep is stage three which is characterized by low frequency, high amplitude delta oscillation of waves $(\sim 1 \mathrm{~Hz})$ known as slow wave sleep (SWS). SWS is thought to relieve sleep pressure and coincides with the timing of peak growth hormone secretion alluding to its role in repairing and maintaining the body while also supporting memory via repeated activation of major memory centers [1]. Percentage of REM sleep and REM latency also have been found to decrease with age [12, 22].

In a large study conducted by the National Institute on Aging, researchers found that sleep complaints increased with age, most notably in women [23]. Objective sleep data corroborate these reports, demonstrating measurable changes in sleep physiology in the elderly. For example, PSG and EEG data in older adults with and without sleep disorders suggest a reduced proportion of deeper, restorative SWS and REM sleep in older adults, leading to problems such as fragmented sleep and early awakenings [5, 22].

There are many mechanisms that may contribute to these age-related changes in sleep. In the process of aging, there is a general decline in the function of the suprachiasmatic nucleus ( $\mathrm{SCN}$ ), which is responsible for regulating 24-h circadian rhythms [24]. The SCN receives light information, subsequently synchronizing neuronal clocks to the light signals and transmits the information to tissues and organs [25]. Changes in circadian rhythms result from this SCN degeneration including phase advance, reduced amplitude seen by changes in body temperature and secretion of hormones such as melatonin and cortisol, a decreased ability for adjusting to phase-shifting, and changes in sleep homeostasis and neuroendocrine function [24]. In addition to biological changes, lifestyle changes associated with aging also appear to affect sleep quality. This may include acute and chronic medical (i.e., cardiovascular, metabolic, and gastrointestinal) illnesses, neuropsychiatric diseases (e.g., depression, anxiety, Alzheimer's disease, Parkinson's disease), and/or psychological stressors (e.g., caregiving, hospitalization, bereavement) [26]. Other common causes for sleep problems in the elderly include changes in behavior or routine (e.g., retirement, decreased daily exercise), environmental reasons (e.g., noise, light, temperature, comfortability), and medications [22]. Due to these multiple causes, sleep interventions paired with increased physical activity may prove to be beneficial.

In addition to affecting quality of life, excessive sleepiness resulting from disordered sleep can lead to lasting physical, psychological, and cognitive effects. Sleep apnea, restless legs syndrome (RLS), REM behavior disorder, and insomnia are 
some of the most prevalent sleep concerns older adults experience. Insomnia is the most common, with close to $30 \%$ of the elderly reporting experiencing it chronically [7, 27].

Insomnia as a sleep disorder is multifaceted and complex. Currently, many models exist concerning etiology and pathophysiology (see [28] for a full review) with Spielman's 3P behavioral model being among the most widely accepted. At present, insomnia is conceptualized by those in the field in accordance with chronicity (i.e., acute, transient, chronic), type (e.g., psychophysiologic, due to medical/psychiatric illness, or idiopathic), and subtype (i.e., initial, middle, or late) [28]. In the elderly, sleep-maintenance insomnia and early awakenings are reportedly more common than sleep-onset difficulties [22]. To meet criteria for a clinical diagnosis, a patient must not only experience diminished sleep quantity and/or quality occurring at least three nights a week for a period of three months, but there must also be clinically significant distress or impairment in one or more life domains [29]. While people who experience reduced or inefficient sleep have poorer cognitive performance such as poorer attention, short-term memory, response times, and performance levels, this is particularly concerning in the elderly population as it decreases their physical functioning and quality of life and increases their likelihood of falls, cognitive impairment, and mortality [27].

\section{Interventions for sleep disorders in the elderly}

\section{CBT-I and other non-pharmacological approaches}

Given that pharmacological interventions for sleep disturbance should only be used for short-term management due to side effects and long-term safety concerns [30], it is important for clinicians to become familiar with nonpharmacological treatments for sleep disturbances in the elderly. These treatments have few, if any, adverse consequences, and research suggests these approaches may be effective even in individuals with cognitive impairment [31]. For the purpose of this paper, we will provide a brief overview of several evidence-based non-pharmacological treatments, highlighting key components of each. For a thorough review of recommended sleep treatments in adults, readers are encouraged to examine Table 11 in the article by SchutteRodin and colleagues [32].

Several non-pharmacological approaches to managing insomnia exist. This includes stimulus control therapy (SCT), sleep restriction treatment (SRT), and relaxation training, each of which have been recommended by the American Academy of Sleep Medicine (AASM) [33-35]. In SCT, the goal of treatment is for the patient to reassociate the bed with sleep by following several guidelines aimed at changing behavior (e.g., only go to bed when sleepy, use the bed only for sleeping or sex, leave the bed if you have not fallen asleep within 10-15 min, wake at the same time each morning, and avoid daytime napping). By engaging in these behaviors, stronger cues between the bedroom and sleeping are formed [36]. For older adults, there are some modifications to these instructions including increasing sleep-onset latency to $20 \mathrm{~min}$ and permitting one short nap (i.e., $<30 \mathrm{~min}$ ) scheduled at the same time each day [37, 38]. Research suggests SCT is an effective treatment for late-life insomnia demonstrating moderate to strong effects on older adults' subjective sleep quality. At this time, SCT does not meet criteria to be considered a stand-alone evidence-based treatment, mainly due to the lack of research exploring it in isolation from CBT-I [39]. Consistent with SCT is SRT which aims to limit total time spent in bed to match sleep ability and sleep opportunity, as derived from sleep diary data, with the purpose being to use homeostatic sleep drive to increase sleep consolidation [40]. Studies examining the efficacy of SRT in older adults show positive results, with particular improvements in self-reported SL and WASO. Notably, the findings of SRT in older adults are so efficacious that it meets American Psychological Association requirements to be considered an EvidenceBased Treatment for late-life insomnia [39]. Relaxation training (e.g., progressive muscle relaxation, guided imagery, breathing training) has also shown to be an effective strategy to combat sleep difficulties in the elderly. In their study of older adults ( $\geq 60$ years), Halpern et al. [41] found that relaxation through yoga significantly improved selfreported sleep quality, efficiency, latency, and duration, in addition to mood concerns.

Cognitive-behavioral therapy for insomnia (CBT-I) has recently been recommended as the first line of treatment by the AASM and National Institute of Health for its strong empirical support demonstrating effectiveness. CBT-I is a brief form of structured treatment (i.e., 6-10 sessions) that helps patients identify maladaptive behaviors and cognitions causing or worsening sleep problems by using a combination of cognitive therapy (i.e., challenging dysfunctional thoughts surrounding sleep) and behavioral therapy (e.g., stimulus control, sleep restriction; see above). Randomized control trials, literature reviews, and meta-analyses have concluded that CBT-I is particularly efficacious for treating insomnia in older adults with findings highlighting superiority over other treatments (pharmacological and non-pharmacological) for both shortand long-term management [42, 43]. For example, Silversten et al. [42] compared CBT-I, Zoplicone (a nonbenzodiazepine prescription sedative), and placebo on a sample of 46 older adults diagnosed with chronic insomnia. They found that in just 6 weeks, patients receiving CBT-I reported more improvements in sleep efficiency, slow-wave sleep, middle of the night awakenings compared to those receiving pharmacological treatment or placebo. These findings 
remained even at 6 months, with patients receiving CBT-I reporting greater improvements in sleep efficiency than those receiving Zoplicone [42]. There is evidence that CBT-I or variants may be efficacious in as few as two sessions for aging adults [44].

\section{Exercise as a sleep intervention}

\section{Exercise as a stand alone intervention}

The relationship between sleep and exercise has been examined in detail. Exercise is commonly used as a measure to improve sleep quality in older adults suffering from insomnia and other sleep-related complications. It has been hypothesized that difficulty sleeping may be exacerbated by increased inflammation in older adults with moderate exercise training recommended as an effective treatment modality [45]. Previous research has shown that elderly participants performing moderate training for $60 \mathrm{~min}$ per day at a rate of 3 days per week over a period of 24 weeks resulted in an increased aerobic capacity parameter and quality of life as well as decreased REM latency and time spent awake [45]. One limitation of this study was that only males were included as participants. Certain types of exercise may be more beneficial with regard to improving sleep quality in patients with insomnia and others suffering from sleep ailments [46-48]. For reference, intensity of aerobic physical activity can be measured in multiple ways (maximal oxygen consumption [\%VO2max], metabolic equivalents [METs], kilocalories [Kcals]) but is commonly and most easily obtained by measuring percentage of maximum heart rate (\% HRmax). Thus, light intensity physical activity is characterized as between 57 and $63 \%$ of maximum heart rate, moderate is $64-76 \%$ of maximum heart rate, and vigorous falls between 77 and 95\%. Maximum heart rate cannot always be maintained directly due to health conditions. However, as a general rule, maximum heart rate can be determined by the use of the equation 220 - age [49].

There is some evidence that moderate intensity levels of aerobic physical activity produce the best results for improving sleep quality [48-50]. For instance, Reid and colleagues [48] found that participants who exercised for three days a week for an average of about $30 \mathrm{~min}$ resulted in improved mood (based on the Center for Epidemiologic Studies Depression [CES-D]), subjective sleep quality (based on the Pittsburgh Sleep Quality Index [PSQI]), and overall quality of life (based on the 36-Item Short Form Health Survey [SF-36]) in older adults with insomnia. Participation in moderate aerobic activity has resulted in improved subjective report of sleep quality when compared to non-physical activity endeavors within the elderly population, with improvements on the PSQI [51] and Epworth Sleepiness Scale (ESS) [52] scores.
In addition, improvements in symptoms of depression based on CES-D scores [53] were reported in one study. When controlling for CES-D scores, it was determined that the beneficial effects of moderate aerobic physical activity on sleep (reported on the PSQI) were independent of the diminished symptoms of depression of participants [48]. In addition, in another study when moderate-intensity aerobic exercise (MAE) was compared to high-intensity aerobic exercise (HAE) and moderate-intensity resistance training (MRE), it was concluded that MAE comparatively yielded more significant results for sleep quality [50]. MAE may have yielded more significant results because it may have been easier to maintain and perform for longer periods than HAE, although this is speculatory at this point. Likewise, there is the possibility of a complex relationship between level of physical fitness and ability to perform HAE (among a primarily sedentary population that was used in the study).

While in general it is assumed that exercise results in improved sleep quality, some studies suggest that sleep quality affects next-day aerobic exercise performance [47]. This was studied with 11 women, mean age 61, with insomnia. They participated in $30 \mathrm{~min}$ of aerobic physical activity 3 times a week for 16 weeks, demonstrating that SL is negatively correlated with next day exercise. These findings provide alternate strategies and a different perspective on the relationship between sleep quality and exercise, indicating that improving sleep would encourage exercise participation the following day [47]. It should be noted that many studies examining sleep and exercise are correlative and do not determine causality. The relationship is obviously complicated. One study, with participants with a mean age of 64 , observed that longer SL produces shorter next day exercise participation, in addition to displaying a positive association between total sleep time (TST) and next day exercise. In addition to these findings, it was also determined that participants who took part in more physical activity reported less wake time during the night. In contrast to the study produced by Baron and colleagues [47], the amount of time it took to fall asleep was not significantly changed with fluctuations in physical activity in other studies. Important demographic notations include a sample size that was majority female $(83.5 \%)$ and 41 out of the 79 participants already led an active lifestyle [54].

With regard to putative effects of resistance training on sleep in elderly populations, more research is needed. A 6month resistance training study with 8 previously sedentary individuals with a mean age of 78 , originally showed improvements on the PSQI score at the 3-month mark of the participants' training. However, upon reaching the 6-month mark the PSQI scores were once again elevated. The authors speculated that this increase in the PSQI score may have been due to the slowing down of strength gains. However, the subject pool was not large enough to draw conclusions and possibly underpowered [55]. A 2014 and 2018 study determined 
that resistance training with older adults did not alter sleep quality in same day sleep. However, resistance training may improve sleep over time with chronic consistent resistance training, specifically sleep apnea in older adults. In addition, the improvements from resistance training are not at the cost of sleep disruption [56, 57]. A 2014 study conducted by Herrick et al. was able to determine positive effects of resistance training in sleep and sleep-related disorders [56]. However, due to averaging PSG results, the 2014 study could not be concluded whether or not an exercise bout would improve upon or disrupt same-day sleep. Thus, a follow-up 2018 study was performed following the same methods without averaging the two PSG scores (one taken the night following resistance training and one on a night not following resistance training [57]). As there is currently no definitive answer with regard to the effects resistance training has on sleep, prescribing aerobic physical activity is generally safer. However, clinically speaking, exercise is not commonly prescribed as a stand-alone intervention for sleep or any ailments for that matter [55].

Given that immune functioning and sleep are tied, it has been theorized that moderate exercise may reduce resting plasma concentrations of pro-inflammatory cytokines and increase anti-inflammatory cytokines, leading to improved overall quality of sleep [58]. Additionally, considering sleep disturbances in older adults are usually multifactorial, interventions that target sleep as well as lifestyle (e.g., exercise) may prove to be beneficial. This combination may assist those who are more sedentary and less engaged in daytime physical and social activities, which are known to impact sleep homeostasis and circadian regulation [59]. Figure 1 provides a proposed and initial explanatory model for the relationship between exercise and sleep. The model also includes potential effects of exercise on psychological variables such as anxiety and depression. At present, the model should be considered as "exploratory" and provides a framework for future research.

\section{Combination of exercise and CBT-I}

CBT-I interventions combined with exercise have yet to be thoroughly examined. In one study that examined patients with cancer and insomnia, mean age of 55, it was concluded that both exercise and CBT-I interventions produced benefits and improvements in sleep. However, CBT-I was superior to aerobic exercise in that it resulted in significantly different scores on the Insomnia Severity Index (ISI) $[60,61]$. Alternatively, aerobic exercise can produce many other added benefits, such as improvements in anxiety, depression, and overall quality of life [62]. Given that results were seen in both exercise and CBT-I individually, one possibility would be to include exercise in the behavioral aspect of CBT-I treatment in order to maximize results in participating patients. One such study included physical activity, light therapy, and CBT-I in order to treat patients with insomnia. The researchers
Fig. 1 Theoretical model of the relationship between moderate exercise and improved sleep function

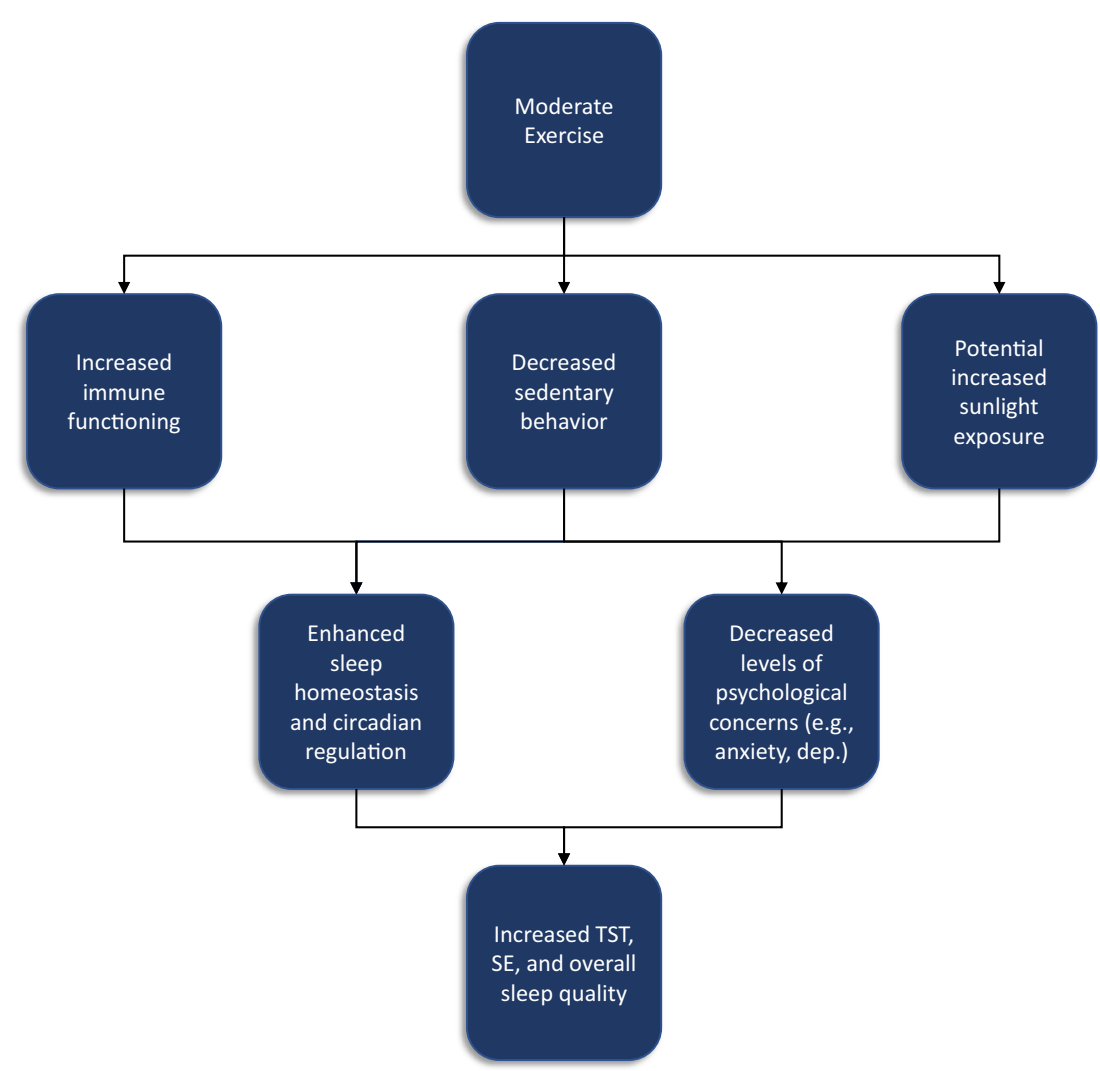


concluded that the treatment was effective in improving sleep latency and quality as well as reduced daytime sleepiness. Participants had an average age of 66 years and were already taking sleep medication prior to starting the trial, making it difficult to interpret effectiveness without the use of medication [63]. To the best of our knowledge, no study has examined the combination of CBT-I and exercise with patients diagnosed with insomnia in an elderly population, leaving this an area of study open to research.

At this time, there are currently no large scale randomized control trials (RCTs) with appropriate controls that have examined the effects of a combination of CBT-I and exercise on sleep disorders in an older adult population. However, there are several studies that have examined both CBT-I and exercise separately in the same population. For example, Guthrie and colleagues [64] examined the effectiveness of several different forms of treatment for nocturnal hot flashes, including yoga, CBT-I, exercise, and escitalopram. The greatest reduction in insomnia symptoms was observed in the CBT-I group (as measured by the ISI and PSQI). However, the aerobic exercise group showed the greatest reduction in symptoms for a non-prescription treatment option. The study was limited in that it did not explore any unique effects on sleep symptoms in the exercise group compared to the CBT-I group.

\section{Future directions and conclusions}

As discussed, sleep quality often declines in the elderly (when using a number of sleep parameters as markers), and aging is associated with increased incidence of insomnia. Pharmacological interventions are at times effective, particularly with short-term use. However, these interventions are often overutilized, and may result in untoward side effects or other downstream effects that diminish sleep quality. There is evidence that non-pharmacological interventions such as CBT-I (or variants of this modality) are effective, sometimes in as few as two sessions among the elderly [44]. There is also evidence that exercise may improve sleep in the elderly. To date, data support the notion that aerobic exercise is beneficial with some aspects of sleep, with less data to support a relationship between resistance training and improved sleep. Varasse, Li, and Gooneratne [65] conclude that there is evidence that exercise as a sleep intervention may be effective and safe, but that future research is needed. At present, there is a lack of controlled investigation or RCTs that examine the potential effects of the combination of behavioral sleep interventions and exercise for elderly individuals who may have sleep disorders. The putative connection between behavioral intervention for sleep and exercise is intuitive to some extent. Yet, there are certain challenges in this course of research in that aging adults tend to engage in less exercise [65]. Future studies may also address the efficacy of combined CBT-I and exercise, disentangle the unique variance that these interventions may contribute to improved sleep, and explore the variables (and associated relationships) in the model presented in Fig. 1. Finally, given mobility and travel restrictions experienced by some aging adults, as well as the current limitations placed on face-to-face interventions during the COVID-19 pandemic, further exploration of computerized delivery and/ or video conference delivery of these interventions is warranted. To date, there is evidence that computerized delivery of CBT-I as a form of self-help may be effective [66]. Likewise, there is evidence that CBT-I teletherapy (or telemedicine) is efficacious in comparison to face-to-face interventions [67]. It is conceivable that such combined tele-protocols including CBT-I and exercise could be advanced and examined in the future.

\section{Appendix. Description of Relevant Sleep Terms}

SWS - slow wave sleep is the deepest phase of non-rapid eye movement (NREM) sleep, and is characterized by delta waves, occurring in stage 3 of sleep (previously 3-4).

REM - rapid eye movement is distinguishable by random and/or rapid movement of the eyes, accompanied with low muscle tone throughout the body

NREM - Non-rapid eye movement consists of sleep stages 1-3 (formerly known as stages 1-4)

TST - total sleep time is the total amount of time spent asleep

SE - sleep efficiency - a percentage derived from total sleep time (minus sleep latency and wake after sleep onset), divided by the total time spent in bed

SL - sleep latency refers to the time that it takes to fall asleep

WASO - wake after sleep onset is the time spent awake after initially falling asleep

EMA - early morning awakening refers to waking up earlier than one intended without the ability to fall back to sleep as desired

K-Complexes - large waves that react to external stimuli while sleeping and define NREM 2

Funding This study received no funding.

\section{Declarations}

Ethical approval This article does not contain any studies with human participants performed by any of the authors.

Conflict of interest Amy Gencarelli declares that she has no conflict of interest. Anne Sorrell declares that she has no conflict of interest. Cassandra Everhart declares that she has no conflict of interest. Taylor Zurlinden declares that she has no conflict of interest. D. Erik Everhart declares that he has no conflict of interest. 


\section{References}

1. Tubbs AS, Dollish HK, Fernandez F, Grandner MA (2019) The basics of sleep physiology and behavior. In Sleep and health (pp. 310). Academic Press

2. Chattu VK, Gholizadeh M, Shapiro CM (2019) Estimation of costs and cost-effectiveness of detecting undiagnosed sleep disorders among chronic disease patients: a typical gp setting in Toronto. Sleep Med 64:S345-S346. https://doi.org/10.1016/j.sleep.2019. 11.964

3. de Mello MT, Narciso FV, Tufik S, Paiva T, Spence DW, Bahammam AS, Verster JC, Pandi-Perumal SR (2013) Sleep disorders as a cause of motor vehicle collisions. Int J Prev Med 4:246257

4. Noh J, Kim K, Lee JH, Lee Y, Lee B, Kwon YD (2017) Association between sleep duration and injury from falling among older adults: a cross-sectional analysis of Korean community health survey data. Yonsei Med J 58:1222-1228. https://doi.org/10.3349/ ymj.2017.58.6.1222

5. Espiritu JR (2008) Aging-related sleep changes. Clin Geriatr Med 24:1-14. https://doi.org/10.1016/j.cger.2007.08.007

6. Allen J, Morelli V (2012) Aging and exercise. Succes Aging, Issue Clin Geriatr Med-E-Book 27(4):661-671

7. Foley DJ, Monjan A, Simonsick EM, Wallace RB, Blazer DG (1999) Incidence and remission of insomnia among elderly adults: an epidemiologic study of 6,800 persons over three years. Sleep 22(Suppl 2):S366-S372

8. Montgomery P (2002) Treatments for sleep problems in elderly people. BMJ 325(7372):1049. https://doi.org/10.1136/bmj.325. 7372.1049

9. Patel D, Steinberg J, Patel P (2018) Insomnia in the elderly: a review. J Clin Sleep Med 14:1017-1024. https://doi.org/10.5664/ jesm. 7172

10. Cirelli C (2012) Brain plasticity, sleep and aging. Gerontology 58: 441-445. https://doi.org/10.1159/000336149

11. Dijk D-J, Duffy JF, Czeisler CA (2000) Contribution of circadian physiology and sleep homeostasis to age-related changes in human sleep. Chronobiol Int 3:285-311. https://doi.org/10.1081/CBI100101049

12. Ohayon MM, Carskadon MA, Guilleminault C, Vitiello MV (2004) Meta-analysis of quantitative sleep parameters from childhood to old age in healthy individuals: developing normative sleep values across the human lifespan. Sleep 17:1255-1273. https://doi. org/10.1093/sleep/27.7.1255

13. Scullin MK (2017) Do older adults need sleep? A review of neuroimaging, sleep, and aging studies. Curr Sleep Med Rep 3(3):204214. https://doi.org/10.1007/s40675-017-0086-z

14. Loomis AL, Harvey EN, Hobart G (1935) Potential rhythms of the cerebral cortex during sleep. Science. 81:597-598. https://doi.org/ 10.1126/science.81.2111.597

15. Tamaki M, Nittono H, Hayashi M, Hori T (2005) Examination of the first-night effect during the sleep-onset period. Sleep 28(2):195202. https://doi.org/10.1093/sleep/28.2.195

16. Crowley K, Trinder J, Kim Y, Carrington M, Colrain IM (2002) The effects of normal aging on sleep spindle and K-complex production. Clin Neurophysiol 113(10):1615-1622. https://doi.org/10. 1016/s1388-2457(02)00237-7

17. Martin N, Lafortune M, Godbout J, Barakat M, Robillard R, Poirier G, Bastien C, Carrier J (2013) Topography of age-related changes in sleep spindles. Neurobiol Aging 34:468-476. https://doi.org/10. 1016/j.neurobiolaging.2012.05.020

18. Nelson PT, Abner EL, Scheff SW, Schmitt FA, Kryscio RJ, Jicha GA, Smith CD, Patel E, Markesbery WR (2009) Alzheimer's-type neuropathology in the precuneus is not increased relative to other areas of neocortex across a range of cognitive impairment. Neurosci Lett 450:336-339. https://doi.org/10.1016/j.neulet.2008.11.006

19. Clawson BC, Durkin J, Aton SJ (2016) Form and function of sleep spindles across the lifespan. Neural Plasticity 6936381. https://doi. org/10.1155/2016/6936381

20. Resnick SM, Pham DL, Kraut MA, Zonderman AB, Davatzikos C (2003) Longitudinal magnetic resonance imaging studies of older adults: a shrinking brain. J Neurosci 23(8):3295-3301. https://doi. org/10.1523/JNEUROSCI.23-08-03295.2003

21. Thambisetty M, Wan J, Carass A, An Y, Prince JL, Resnick SM (2010) Longitudinal changes in cortical thickness associated with normal aging. Neuroimage 52(4):1215-1223. https://doi.org/10. 1016/j.neuroimage.2010.04.258

22. Suzuki K, Miyamoto M, Hirata K (2017) Sleep disorders in the elderly: diagnosis and management. J Gen Fam Med 18(2):61-71. https://doi.org/10.1002/jgf2.27

23. Foley DJ, Monjan AA, Brown SL, Simonsick EM, Wallace RB, Blazer DG (1995) Sleep complaints among elderly persons: an epidemiologic study of three communities. Sleep 18:425-443. https://doi.org/10.1093/sleep/18.6.425

24. Li J, Vitiello MV, Gooneratne NS (2018) Sleep in normal aging. Sleep Med Clin 13(1):1-11. https://doi.org/10.1016/j.jsmc.2017. 09.001

25. Albrecht U, Ripperger JA (2018) Circadian clocks and sleep: impact of rhythmic metabolism and waste clearance on the brain. Trends Neurosci 41(10):677-688

26. Vaz Fragoso CA, Gill TM (2007) Sleep complaints in communityliving older persons: a multifactorial geriatric syndrome. J Am Geriatr Soc 55(11):1853-1866. https://doi.org/10.1111/j.15325415.2007.01399.x

27. Roepke SK, Ancoli-Israel S (2010) Sleep disorders in the elderly. Indian J Med Res 131:302-310. https://doi.org/10.1016/S13899457(00)00083-6

28. Perlis M, Shaw P, Cano G, Espie C (2011) Models of insomnia. In: Kryger MH, Roth T, Dement WC (eds) Principles and Practices of Sleep Medicine. Elsevier, Philadelphia, pp 850-865

29. American Psychiatric Association (2013) Diagnostic and statistical manual of mental disorders, 5th edn. APA, Arlington

30. Brewster GS, Riegel B, Gehrman PR (2018) Insomnia in the older adult. Sleep Med Clin 13(1):13-19. https://doi.org/10.1016/j.jsmc. 2017.09.002

31. Shub D, Darvishi R, Kunik ME (2009) Non-pharmacologic treatment of insomnia in persons with dementia. Geriatrics 64:22

32. Schutte-Rodin S, Broch L, Buysse D, Dorsey C, Sateia M (2008) Clinical guideline for the evaluation and management of chronic insomnia in adults. J Clin Sleep Med 4:487-504

33. Sateia MJ, Buysse DJ, Krystal AD, Neubauer DN, Heald JL (2017) Clinical practice guideline for the pharmacologic treatment of chronic insomnia in adults: an American Academy of Sleep Medicine clinical practice guideline. J Clin Sleep Med 13(2):307349. https://doi.org/10.5664/jcsm.6470

34. Montgomery P, Dennis J (2004) A systematic review of nonpharmacological therapies for sleep problems in later life. Sleep Med Rev 8:47-62. https://doi.org/10.1016/S1087-0792(03)000261

35. Morgenthaler T, Kramer M, Alessi C, Friedman L, Boehlecke B, Brown T, Coleman J, Kapur V, Lee-Chiong T, Owens J, Pancer J, Swick T, American Academy of Sleep Medicine (2006) Practice parameters for the psychological and behavioral treatment of insomnia: an update. An American academy of sleep medicine report. Sleep 29(11):1415-1419

36. Siebern AT, Suh S, Nowakowski S (2012) Non-pharmacological treatment of insomnia. Neurotherapeutics 9(4):717-727. https://doi. org/10.1007/s13311-012-0142-9 
37. Joshi S (2008) Nonpharmacologic Therapy for Insomnia in the Elderly. Clin Geriatr Med 24:107-119. https://doi.org/10.1016/j. cger.2007.08.005

38. Bootzin RR, Perlis ML (2011) Stimulus control therapy. In: Perlis ML, Aloia M, Kuhn B (eds) Behaviral treatments for sleep disorders: a comprehensive primer of behavioral sleep medicine interventions. Elsevier, Boston, pp 21-30

39. McCrae CS, Dzierzewski JM, Kay D (2009) Treatment of late-life insomnia. Sleep Med Clin 4(4):593-604. https://doi.org/10.1016/j. jsmc.2009.07.006

40. Spielman AJ, Yang C, Glovinsky PB Sleep restriction therapy. In: Kuhn, Aloia, Perlis (eds) Behavioral treatments for sleep disorders: a comprehensive primer of behavioral sleep medicine interventions. Elsevier, Philadelphia, pp 9-19

41. Halpern J, Cohen M, Kennedy G, Reece J, Cahan C, Baharav A (2014) Yoga for improving sleep quality and quality of life for older adults. Altern Ther Health Med 20(3):37-46

42. Sivertsen B, Omvik S, Pallesen S, Bjorvatn B, Havik OE, Kvale G, Nielsen GH, Nordhus IH (2006) Cognitive behavioral therapy vs zopiclone for treatment of chronic primary insomnia in older adults: a randomized controlled trial. JAMA. 295(24):2851-2858. https:// doi.org/10.1001/jama.295.24.2851

43. Mitchell MD, Gehrman P, Perlis M, Umscheid CA (2012) Comparative effectiveness of cognitive behavioral therapy for insomnia: a systematic review. BMC Fam Pract 13:40. https://doi. org/10.1186/1471-2296-13-40

44. Buysse DJ, Germain A, Moul DE, Franzen PL, Brar LK, Fletcher ME, Begley A, Houck PR, Mazumdar S, Reynolds CF III, Monk TH (2011) Efficacy of brief behavioral treatment for chronic insomnia in older adults. Arch Intern Med 171(10):887-895. https://doi. org/10.1001/archinternmed.2010.535

45. Santos RD, Viana VAR, Boscolo RA, Marques VG, Santana MGD, Lira FSD et al (2012) Moderate exercise training modulates cytokine profile and sleep in elderly people. Cytokine 60(3):731735

46. Banno M, Harada $\mathrm{Y}$, Taniguchi M, Tobita R, Tsujimoto H, Tsujimoto Y, Kataoka Y, Noda A (2018) Exercise can improve sleep quality: a systematic review and meta-analysis. PeerJ 6: e5172. https://doi.org/10.7717/peerj.5172

47. Baron KG, Reid KJ, Zee PC (2013) Exercise to improve sleep in insomnia: exploration of the bidirectional effects. J Clin Sleep Med 9(8):819-824. https://doi.org/10.5664/jcsm.2930

48. Reid KJ, Baron KG, Lu B, Naylor E, Wolfe L, Zee PC (2010) Aerobic exercise improves self-reported sleep and quality of life in older adults with insomnia. Sleep Med 11(9):934-940. https:// doi.org/10.1016/j.sleep.2010.04.014

49. ACSM (2018) ACSM's guidelines for exercise testing and prescription, 10th edn. Wolters Kluwer, Philadelphia

50. Passos GS, Poyares D, Santana MG, Garbuio SA, Tufik S, Mello MT (2010) Effect of acute physical exercise on patients with chronic primary insomnia. J Clin Sleep Med 6(3):270-275

51. Buysse DJ, Reynolds CF, Monk TH, Berman SR, Kupfer DJ (1989) The Pittsburgh Sleep Quality Index: a new instrument for psychiatric practice and research. Psychiatry Res 28:193-213

52. Johns MW (1991) A new method for measuring daytime sleepiness: The Epworth Sleepiness Scale. Sleep: J Sleep Res Sleep Med 14:540-545
53. Radloff LS (1977) The CES-D Scale: A self-report depression scale for research in the general population. Appl Psychol Meas 1:385401. https://doi.org/10.1177/014662167700100306

54. Dzierzewski JM, Buman MP, Giacobbi PR et al (2014) Exercise and sleep in community-dwelling older adults: evidence for a reciprocal relationship. J Sleep Res 23:61-68. https://doi.org/10.1111/ jsr. 12078

55. Ferris LT, Williams JS, Shen CL, O'Keefe KA, Hale KB (2005) Resistance training improves sleep quality in older adults: A pilot study. J Sports Sci Med 4:354-360

56. Herrick JE, Bliwise DL, Puri S, Rogers S, Richards KC (2014) Strength training and light physical activity reduces the apneahypopnea index in institutionalized older adults. J Am Med Dir Assoc 15:844-846. https://doi.org/10.1016/j.jamda.2014.08.006

57. Herrick JE, Puri S, Richards KC (2018) Resistance training does not alter same-day sleep architecture in institutionalized older adults. J Sleep Red 27(4):e12590. https://doi.org/10.1111/jsr.12590

58. Kapsimalis F, Basta M, Varouchakis G, Gourgoulianis K, Vgontzas A, Kryger M (2008) Cytokines and pathological sleep. Sleep Med 9(6):603-614

59. Li J, Yang B, Varrasse M, Li K (2018) Sleep among long-term care residents in China: a narrative review of literature. Clin Nurs Res 27(1):35-60. https://doi.org/10.1177/1054773816673175

60. Morin CM, Belleville G, Bélanger L, Ivers H (2011) The insomnia severity index: psychometric indicators to detect insomnia cases and evaluate treatment response. Sleep 34(5):601-608

61. Mercier J, Ivers H, Savard J (2018) A non-inferiority randomized controlled trial comparing a home-based aerobic exercise program to a self-administered cognitive-behavioral therapy for insomnia in cancer patients. Sleep 41(10). https://doi.org/10.1093/sleep/zsy149

62. Berger BG, Motl RW (2000) Exercise and mood: A selective review and synthesis of research employing the profile of mood states. J Appl Sport Psychol 12:69-92. https://doi.org/10.1080/ 10413200008404214

63. Richter K, Myllymaeki J, Scharold-Schaefer S, Tomova I, Mayrer R, Niklewski G (2014) Treating comorbid insomnia in older adults via cognitive-behavioural treatment, bright light and exercise. Health 6:960-968. https://doi.org/10.4236/health.2014.610121

64. Guthrie KA, Larson JC, Ensrud KE, Anderson GL, Carpenter JS, Freeman EW, Joffe H, LaCroix AZ, Manson JAE, Morin CM, Newton KM, Otte J, Reed SD, McCurry SM (2018) Effects of pharmacologic and nonpharmacologic interventions on insomnia symptoms and self-reported sleep quality in women with hot flashes: a pooled analysis of individual participant data from four MsFLASH trials. Sleep 41. https://doi.org/10.1093/sleep/zsx190

65. Varrasse M, Li J, Gooneratne N (2015) Exercise and sleep in community-dwelling older adults. Curr Sleep Med Rep 1:232240. https://doi.org/10.1007/s40675-015-0028-6

66. Cheng SK, Dizon J (2012) Computerised cognitive behavioural therapy for insomnia: a systematic review and meta-analysis. Psychother Psychosom 81:206-216. https://doi.org/10.1159/ 000335379

67. Hsieh C, Rezayat T, Zeidler MR (2020) Telemedicine and the management of insomnia. Sleep Med Clin 15:383-390. https://doi.org/ 10.1016/j.jsmc.2020.05.004

Publisher's note Springer Nature remains neutral with regard to jurisdictional claims in published maps and institutional affiliations. 